\title{
When Is Watchful Waiting Warranted? Advances in Soft Tissue Sarcoma-An Editorial Commentary
}

\author{
Carolyn Nessim, MSc, MD, FRCSC, FACS \\ Department of Surgery, The Ottawa Hospital Research Institute, Ottawa, ON, Canada
}

\section{CURRENT STANDARD OF CARE}

Traditionally, when a patient presents with an unplanned excision (UE) for an extremity or truncal soft tissue sarcoma (STS), the recommendation has been a re-resection of the tumor bed with or without neoadjuvant or adjuvant radiation and with or without chemotherapy, depending on the histological subtype. This recommendation has long since held as often margins of these UEs are difficult to assess as it is not always clear if the patient underwent an $\mathrm{R} 0$ resection (i.e. wide resection), $\mathrm{R} 1$ resection (i.e. visibly macroscopically complete marginal resection with only microscopically positive margins on pathology), or an R2 resection (i.e. a partial, piecemeal, or incisional resection with grossly macroscopically positive margins). This is especially true in patients who do not exhibit obvious residual palpable disease on clinical examination. It is clear from several retrospective studies to date that an R2 resection has a worse overall oncologic outcome when compared with an $\mathrm{R} 0$ or $\mathrm{R} 1$ resection, and that these patients need a re-resection. What is unclear is the true impact of an R1 resection on overall and distant diseasefree survival (DDFS), and if re-resection, when there is no visible residual tumor, is systematically warranted.

(C) Society of Surgical Oncology 2019

First Received: 29 May 2019;

Published Online: 17 June 2019

C. Nessim, MSc, MD, FRCSC, FACS

e-mail: cnessim@toh.ca
THE ROLE OF MARGIN STATUS

Many studies have shown that only a minority of patients who have an R1 resection actually recur. One of the largest studies evaluating margin status in STS looked at 2084 patients, of whom only $22 \%$ had a positive margin. Of these R1 resections, only $28 \%$ recurred; however, more importantly, $72 \%$ never recurred. ${ }^{1}$ Furthermore, the risk of recurrence for those with an R0 resection was still $15 \%$. This study showed that a positive margin increased the risk of recurrence twofold compared with an R0 resection, and also increased disease-related death. However, these retrospective studies can have inherent bias, and although there may be an association with R1 resections, and potentially a worse overall outcome, it cannot be confirmed as causal and it is difficult to ensure that all confounding factors have been accounted for.

\section{THE ROLE OF RE-RESECTION}

A multitude of retrospective studies have shown there may be benefit to re-resection after a UE. In one of the largest studies by Zagars et al. ${ }^{2} 666$ patients with UE were evaluated; 295 underwent re-resection (46\% were found to have residual tumor, the majority being microscopic) and were compared with 377 patients who only had radiation. This study showed that local control, DFS, and DDFS were all better in the group with re-resection; however, again, there was an inherent selection bias in this retrospective study as the two populations were not equal. Those not having a re-resection had worse histologies with deeper and larger tumors that could not be re-resected, thus underlining the difference in biology between the two groups. On the contrary, other studies have shown no difference in local recurrence or overall survival when comparing planned primary wide resection with re-resection after UE performed in a referral sarcoma center, ${ }^{3,4}$ stating that outcome may be salvaged after a UE. 
Nonetheless, do we really know the true outcome without re-resection? It is important to note that in another large study comparing patients undergoing a primary planned wide resection versus a UE, there were quite significant differences between the two populations, with the UE tumors being more superficial in the subcutaneous tissues as opposed to being deep to fascia, as well as being smaller and with lower-grade histologies, thus inherently having a better biology and hence better outcome overall when compared with the planned resection group. ${ }^{4}$ Thus, histological grade, size, and depth of tumor may trump the $\mathrm{R} 1$ resection.

\section{IS ROUTINE RE-RESECTION WARRANTED?}

Re-resection does not come without morbidity. To ensure an R0 resection, the re-resection surgery is often more extensive than an initially properly planned wide resection for a known sarcoma. During re-resection surgery, the margins will be wider to potentially account for contamination from the previous operation, and the entire tumor bed needs to be resected based on imaging prior to the UE, when available, hence more likely requiring skin flaps or skin grafts, which has been shown in several studies. ${ }^{4,5}$ Moreover, surgery is often blindly performed in these cases as there is often no palpable tumor at the time of re-resection, and thus the extent of the additional margin that must be taken is not fully known. Interestingly, in the study by Chandrasekar et al. ${ }^{6}$ on 316 re-resected specimens, $59 \%$ had residual tumor. Of these, $12 \%$ still had an R1 resection, of which $46 \%$ recurred. The remaining $47 \%$ had an R0 on re-resection, of which $20 \%$ still recurred. This speaks to the likelihood of tumor biology playing a significant role in the risk of recurrence, more so than the extent of surgery. Wider margins may help some patients, but it is quite difficult to identify which patients will benefit the most from these wider re-resections.

The paper by Decanter et al. ${ }^{7}$ is one the largest more modern studies to date, evaluating 622 patients who had a UE with no visible residual disease. This study shows interesting results using a watchful waiting approach in which 251 patients did not have a re-resection. Despite these patients having larger, more aggressive and deeper tumors than those who had upfront re-resection, in this watchful waiting cohort $40 \%$ never recurred, avoiding a needless operation (this is not an insignificant number of patients). In those who did recur, a salvage operation was always possible, with no effect on patients' overall survival or metastatic-free survival when compared with patients who had upfront re-resection surgery in a specialized sarcoma center. Biology therefore allowed surgeons to select out those patients who may have required surgery, without risking overall survival or outcome, while still providing adequate local control. Moreover, re-resection at the time of recurrence is easier as there is a palpable mass that can be delineated to guide surgical wide margins.

Although not statistically significant, it is important to note that six patients (minority) required an amputation upon recurrence. It is difficult to ascertain if these patients would have initially required amputation if they were offered upfront re-resection, however that is the limitation of this retrospective study. Finally, it is also important to note that when comparing the three groups in the study, those who had a planned wide re-resection surgery after UE in a specialized sarcoma center also had the best outcomes with regard to local recurrence-free survival. However, if local control can still be offered by delayed surgery, with a reasonable functional outcome, only to those who really need it, without worrying about affecting their overall survival, this can lead to a significant change in practice and reduce overall morbidity in this patient population, thus improving quality of care, quality of life, and allowing us to follow the ultimate principle of medicine to 'do no harm'. These results are quite intriguing but remain retrospective in nature, comparing quite different patient populations.

\section{CONCLUSION}

These results are compelling enough to urge sarcoma centers that specialize in these surgeries to collaborate, as in the Transatlantic Australasian Retroperitoneal Sarcoma Working Group (TARPSWG), to potentially run a multicenter randomized clinical trial comparing upfront reresection with watchful waiting (and delayed re-resection when necessary) after a macroscopically complete (R1) UE of a superficial extremity or truncal STS. Using an appropriate watchful waiting surveillance program with serial imaging and clinical examination may be a safe approach in patients with a UE but who are macroscopically complete.

\section{REFERENCES}

1. Stojadinovic A, Leung DH, Hoos A, Jaques DP, Lewis JJ, Brennan MF. Analysis of the prognostic signficance of microscopic margins in 2,084 localized primary adult soft tissue sarcomas. Ann Surg. 2002;235:424-34.

2. Zagars GK, Ballo MT, Pisters PW, Pollock RE, Patel SR, Benjamin RS. Surgical margins and reresection in the management of patients with soft tissue sarcoma using conservative surgery and radiation therapy. Cancer. 2003;97:2544-53.

3. Funovics PT, Vaselic S, Panotopoulos J, Kotz RI, Dominkus M. The impact of re-excision of inadequately resected soft tissue sarcomas on surgical therapy, results, and prognosis: a single institution experience with 682 patients. J Surg Oncol. 2010;102:626-33. 
4. Smolle MA, Tunn PU, Goldenitsch E, et al. The Prognostic Impact of Unplanned Excisions in a Cohort of 728 Soft Tissue Sarcoma Patients: A Multicentre Study. Ann Surg Oncol. 2017;24:1596-605.

5. Potter BK, Adams SC, Pitcher JD Jr, Temple HT. Local recurrence of disease after unplanned excisions of high-grade soft tissue sarcomas. Clin Orthop Relat Res. 2008;466:3093-100.

6. Chandrasekar CR, Wafa H, Grimer RJ, Carter SR, Tillman RM, Abudu A. The effect of an unplanned excision of a soft-tissue sarcoma on prognosis. J Bone Joint Surg B. 2008;90:203-8.
7. Decanter G, Stoeckle E, Honore C, et al. Watch and wait approach for re-excision after unplanned yet macroscopically complete excision of extremity and superficial truncal soft tissue sarcoma is safe and does not affect metastatic risk or amputation rate. Ann Surg Oncol. 2019. In Press.

Publisher's Note Springer Nature remains neutral with regard to jurisdictional claims in published maps and institutional affiliations. 